\title{
LAS CREENCIAS SOBRE OBESIDAD DE ESTUDIANTES DE LA EDUCACIÓN GENERAL BÁSICA
}

\author{
Hilda Patricia Núñez Rivas \\ Investigadora del Instituto Costarricense de Investigación \\ y Enseñanza en Nutrición y Salud (INCIENSA) \\ San José, Costa Rica
}

Recibido 25-VI-2007

Aceptado 12-VIII-2007

\begin{abstract}
Resumen: Se discuten las creencias acerca de la obesidad que poseen los niños y las niñas y sus familiares, producto de una investigación doctoral, cualitativa y etnográfica que se desarrolló en un centro educativo público y urbano de San José, Costa Rica. $\mathrm{Su}$ propósito principal fue comprender en profundidad el desarrollo de la obesidad en la niñez, para lo cual se consideraron tres escenarios: familia, escuela $y$ comunidad. Se espera que este artículo brinde aportes teóricos y prácticos para mejorar la comprensión del desarrollo de la obesidad en la población escolar y la praxis del personal de salud y docente, en relación con la prevención de ésta, mediante el desarrollo de intervenciones educativas adecuadas.
\end{abstract}

Palabras clave: Creencias, obesidad, niñez, familia, escuelas, intervención educativa.
"Hacemos juicios a base de prejuicios. Se hace ciencia a partir de la pre-ciencia. Se hace la demostración apoyándose en la creencia. Desde la creencia piensa el filósofo. Desde la creencia interpreta el científico. Desde la creencia salva y condena el religioso. De la creencia vive el sabio y el ignorante, el bueno y el malo, el súbdito y el gobernante. Por la creencia muere el héroe, mata el fanático; muere el mártir, mata el dogmático; muere el revolucionario, mata el mesiánico."

De La Pienda (1999: 242)

\section{Introducción}

Las consecuencias en la salud del exceso de peso durante la infancia son diversas y el mismo ha sido asociado principalmente con los factores de riesgo clásicos de enfermedad cardiovascular: hipertensión arterial (Figueroa-Colon, Franklin, Lee, Aldridge, Alexander, 1997), dislipidemias (Freedman, Dietz, Srinivasan, Berenson, 1997), síndrome metabólico (Weiss, Dziura, Burgert, 2004; Cook, Weitzman, Auinger, Nguyen, Dietz, 2003), obesidad abdominal (Morrison, Barton, 


\begin{abstract}
This paper is related to the beliefs of the children and their families' obesity. These beliefs are part of the main outcomes of a doctoral thesis, which used the qualitative perspective and ethnographic method. This research was carried out in a public, urban educational center of San José, Costa Rica. The main purpose was to understand in depth the development of the obesity in children. The research considered three contexts: family, school, and community. It is expected that this article offers theoretical and practical contributions to improve the understanding of the development of obesity in elementary school-aged children and the "praxis" of health-educational personnel in the prevention of it by means of the development of appropriate educational interventions.
\end{abstract}

Key words: Beliefs, obesity, childhood, family, school, educative intervention.
Biro, Daniels, Sprecher, 1999), glicemia elevada y diabetes tipo 2. (Pinhas-Hamiel, Dolan, Daniels, Standford, Khoury, Zeitler, 1996)

También, se documenta que el sobrepeso y la obesidad durante la niñez y la adolescencia tienen consecuencias de naturaleza psicosocial en estos grupos; entre éstas: baja autoestima (principalmente en las niñas) (Strauss, 2000) y aislamiento social (Strauss, Pollack, 2003). Se reporta que los niños obesos y las niñas obesas, con frecuencia, son objeto de burla por parte de sus pares con estado nutricional normal. (Janssen, Craig, Boyce, Pickett, 2004)

La prevalencia de obesidad (con sus consecuencias multifactoriales) en el ámbito mundial, así como la esperanza de vida en los países en desarrollo, se están incrementando (WHO, 2000). Sin embargo, la posibilidad de prolongar los años de vida, no dependerá únicamente de la reducción en la mortalidad infantil, sino también de la disminución en las enfermedades crónicas no transmisibles asociadas con la mortalidad prematura (Frenk, Freika, Bobadilla, Stern, Lozano, Sepúlveda, 1991); por lo que una disminución en la prevalencia de obesidad en la niñez, mediante intervenciones educativas multidisciplinarias, podría contribuir a lograr esta meta. (WHO, 2000)

Actualmente, la obesidad y el sobrepeso constituyen una de las enfermedades crónicas más frecuentes en la niñez costarricense. Resultados de la última encuesta nacional de nutrición indican que 14,9\% de la población escolar presenta sobrepeso. (Ministerio de Salud, 1996)

En el 2003, Núñez y colaboradores, llevaron a cabo un estudio para determinar la prevalencia de obesidad en niños y niñas de 7 a 12 años de edad, de 36 escuelas urbanas y rurales de la Gran Área Metropolitana de Costa Rica y reportaron prevalencias de sobrepeso y obesidad de $34,5 \%$ y $26,2 \%$, respectivamente. Se encontró mayor prevalencia de obesidad en la 
población de 7 a 9 años, masculina, de área urbana y de grado socioeconómico mediano. (Núñez, Monge, Elizondo, 2003)

Dichas cifras y en general la situación de salud de la población escolar costarricense, han justificado que recientemente, los equipos gubernamentales de los sectores salud y educación, enfoquen sus políticas y programas hacia la prevención de enfermedades no transmisibles en la niñez y la población joven.

La Política Nacional de Alimentación y Nutrición 2006-2010 y la Política Nacional de Salud 2002-2006 contemplan entre sus estrategias, la promoción de hábitos de alimentación, recreación y actividad física saludables, y la defensa y el fortalecimiento del derecho a la atención integral en salud de la niñez y la población adolescente, con calidad, equidad, universalidad, solidaridad y enfoque de género.

La Comisión Nacional de Transversalidad del Ministerio de Educación Pública de Costa Rica, elaboró el documento "Transversalidad en el Currículo Educativo Costarricense, Relanzamiento de la Educación Costarricense" (MEP, 2004), con el objetivo de contribuir a mejorar el proceso de enseñanza-aprendizaje, mediante el quehacer pedagógico de los docentes.

Ese quehacer se constituye en una guía para generar transformaciones en el ámbito del trabajo de aula y enfrentar los retos que demanda la sociedad actual; lo cual justifica aún más, considerar la obesidad como fenómeno de estudio del ámbito educativo, por ser ésta un problema de grandes dimensiones y repercusiones en la población escolar.

Entre los enfoques y competencias de los temas transversales que propone la Comisión mencionada está la Educación para la Salud, que enfatiza la promoción de estilos de vida saludables en los programas de estudio de la Educación General Básica (EGB). (MEP, 2004)

Además, el Ministerio de Educación Pública cuenta con instancias asesoras en las direcciones regionales, cuyas funciones incluyen la orientación del personal a cargo de los comedores y sodas de las escuelas, en relación con la implementación de menúes balanceados.

\section{Referente teórico}

De acuerdo con los antecedentes mencionados, las temáticas sobre estilos de vida saludables y la prevención de la obesidad en la niñez, han sido consideradas entre los objetivos, los contenidos y los procesos de educación formal de los sectores de salud y educación. No obstante, en la praxis, no se conciben a las niñas y los niños como sujetos socio-históricos, que en forma permanente están experimentando aprendizajes, mediante intensas interacciones sociales y procesos educativos formales y no-formarles, los cuales se recrean en ambientes tan amplios, que engloban los escenarios familiar, escolar y comunitario.

A su vez, las instituciones gubernamentales de ambos sectores dejan de lado que, dichos escenarios reciben las influencias económica, política y cultural de otros ambientes no tan inmediatos a la niñez, tales como, la industria alimentaria, los medios de comunicación y las normas sociales, que de no tener una orientación holística, podrían favorecer el establecimiento de ambientes obesogénicos (Figura 1). Para la niñez, todos los ambientes son construidos socialmente, porque las niñas y los niños no escogen qué comerán ni dónde vivirán (si su barrio o comunidad son o no adecuados para la recreación y la actividad física).

Los programas gubernamentales para la prevención de la obesidad que se desarrollan de acuerdo con el punto de vista etic (desde afuera), se basan en la teoría existente o en una clasificación generalizada y determinada de antemano, y se limitan al marco de referencia del investigador (Díaz, 2006). Esto no debería de ser así; no se trata de codificar regularidades abstractas ni de generalizar entre 


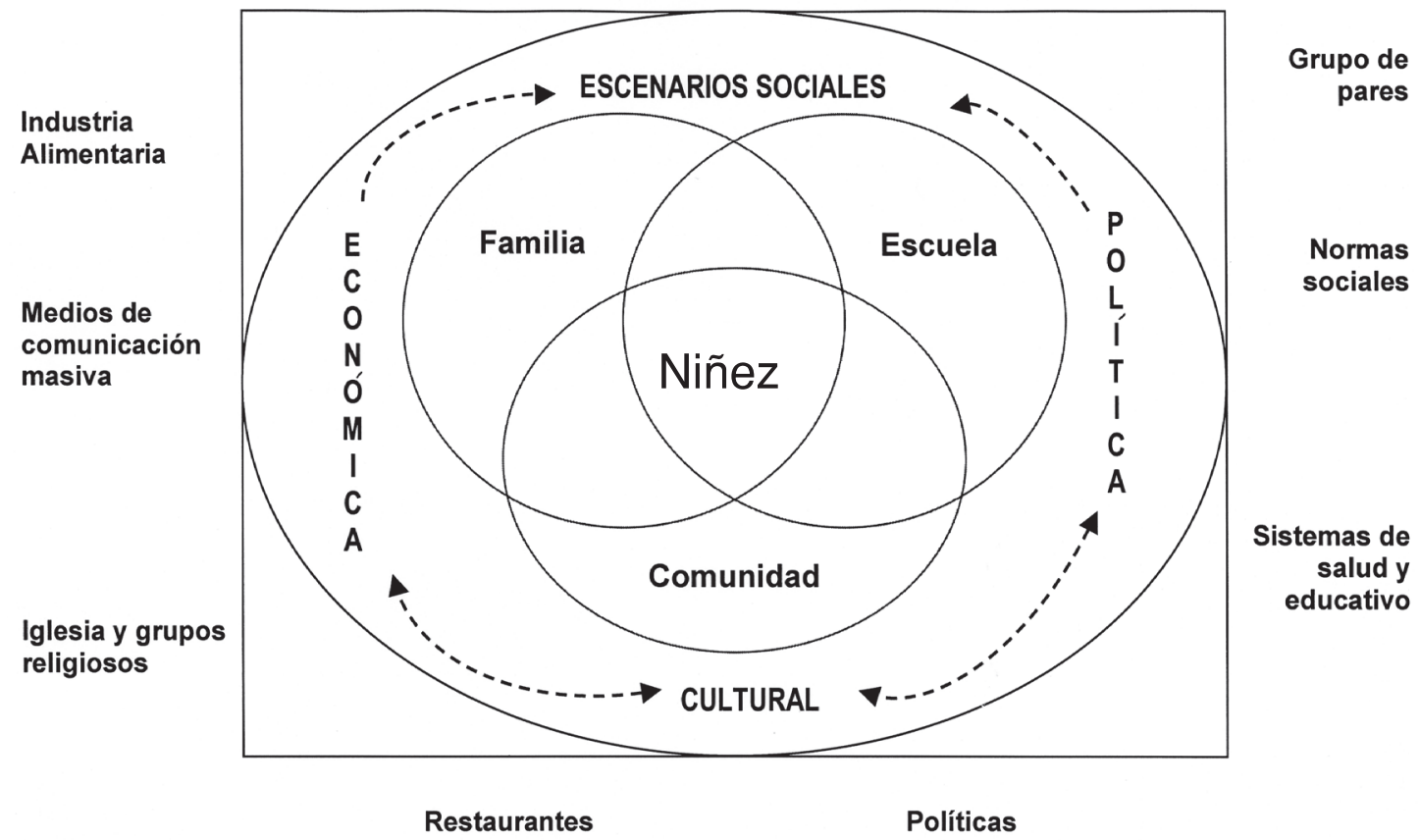

Figura 1. Constituyentes de los ambientes sociales de las niñas y los niños.

casos diversos, más bien, se deben buscar patrones o tendencias dentro de esos mismos casos.

De hecho, Geertz (1973) sostiene que en lugar de comenzar por una serie de observaciones y tratar de ajustar esos casos a una ley que los gobierne, tal inferencia comienza con una serie de presuntos significantes e intentos de situarlos en un marco inteligible.

Por ello, para que los programas gubernamentales de prevención de la obesidad sean integrales y exitosos, deben incluir también el punto de vista emic (desde adentro). Es decir, serán tanto más fructíferos cuanto más compensen estos dos extremos en tensión dialéctica (eticemic). Y serán pobres cuando la perspectiva etic invisibilice el mundo interpretado por los nativos. Asimismo, carecerán de riqueza, cuando el punto de vista emic domine tanto como para carecer de todo valor científico, o sea de todo valor para otros investigadores. (Díaz, 2006)
Entre los aspectos relacionados con la perspectiva emic, en esta investigación cualitativa cobró importancia el estudio de las creencias sobre la obesidad que las niñas y los niños construyen y reconstruyen socialmente en los escenarios familiar, escolar y comunitario. Ello, debido a que previo a esta investigación, en Costa Rica existía un vacío de conocimiento sobre este tema.

Este conocimiento es un insumo imprescindible para el diseño de intervenciones educativas con pertinencia cultural, que busquen la prevención exitosa de la obesidad en la población escolar. Además, se destaca que en el ámbito internacional, la literatura científica sobre esta temática es escasa.

El niño y la niña elaboran distintas representaciones sociales sobre la obesidad, de acuerdo con la influencia (formas de pensar, creencias construidas, etc.) de sus pares, familiares y otras fuentes con las que están en contacto cotidianamente, 
tanto de manera directa como indirecta (Figura 1). Las creencias, al igual que las ideas y las opiniones de las personas son transmitidas y recibidas de generación en generación, sin importar su veracidad.

Carey (1992), plantea que tanto los conceptos como las creencias y las teorías son representaciones mentales que son concebidas como ciertas, y Dewey (1989) afirma que en las creencias se confía lo suficiente como para actuar de acuerdo con ellas, a pesar de que éstas encierren todas las cuestiones sobre las cuales no se dispone de un conocimiento seguro.

De acuerdo con estos supuestos teóricos, se realizó esta investigación doctoral bajo la perspectiva cualitativa y el uso del método etnográfico, en un centro educativo público y urbano de San José, Costa Rica. $\mathrm{Su}$ propósito general fue comprender en profundidad el desarrollo de la obesidad en la niñez, mediante: -el análisis de las creencias sobre obesidad que tienen los niños y las niñas de tercero y cuarto año de la EGB, sus familiares, docentes, el personal a cargo del comedor y la soda escolar; -la exploración del ambiente familiar (hogares) y -el análisis de las políticas (explícitas e implícitas) sobre alimentación y actividad física de la escuela urbana pública, así como de los contenidos educativos de los programas de estudio de la asignatura Educación Física y el desarrollo de las clases de esta asignatura.

En este artículo se discuten parcialmente los hallazgos relacionados con el análisis del sistema de creencias sobre obesidad que tienen los niños y las niñas y sus familiares. El resto de los resultados serán discutidos en otros artículos científicos que serán publicados posteriormente.

Se espera que este artículo brinde aportes teóricos y prácticos para mejorar la comprensión del desarrollo de la obesidad en la población escolar y la praxis del personal de salud y docente, en relación con la prevención de la obesidad, mediante el desarrollo de intervenciones educativas adecuadas.

\section{Metodología}

Tipo de estudio: Estudio cualitativo y transversal que utilizó el método etnográfico para poner en evidencia lo que acontecía cotidianamente en la vida escolar y extra-escolar de las niñas y los niños de tercero y cuarto año matriculados en una escuela pública y urbana de San José, Costa Rica; con el fin de aportar información sobre sus creencias acerca de la obesidad y sus prácticas de alimentación, recreación y actividad física, así como las de sus familias, docentes y personal administrativo del centro educativo.

Se enmarca como estudio de caso, porque se realizó un análisis en profundidad de todo lo que ocurría en la escuela, hogares y barrios de esos estudiantes, en relación con el desarrollo de la obesidad, los aspectos evidentes y los elementos implícitos y simbólicos que subyacen en las prácticas cotidianas y las interacciones sociales entre los sujetos de estudio.

Población de estudio: La selección de los sujetos se hizo de acuerdo con la curiosidad epistemológica de la autora y la cooperación voluntaria de algunos informantes claves, que se obtuvo durante el trabajo de campo, el cual tuvo una duración de 11 meses. Quedó conformado por once niñas obesas (8 de cuarto año y tres de tercer año) y seis niños obesos (cinco de cuarto año y uno de tercer año); quince madres y un padre (no se logró la cooperación de los progenitores de una niña de cuarto año); doce docentes; dos personas a cargo del servicio del comedor de la escuela, la concesionaria a cargo de la soda escolar; una integrante de la Junta de Educación, una persona a cargo de la limpieza de la escuela, el guarda que tiene a cargo la seguridad del centro educativo, y por 235 niñas y 225 niños de segundo a sexto año matriculados en la escuela, caso de estudio.

Debido a que en este artículo se discuten parcialmente los hallazgos relacionados con únicamente el sistema de creencias 
que acerca de la obesidad tienen los niños, las niñas y sus familiares; las informaciones del resto de los sujetos de estudio serán discutidas en otros artículos científicos que se publicarán posteriormente.

Técnicas e instrumentos: Si-guiendo el planteamiento de Rodríguez, Gil y García (1996), se utilizaron distintas técnicas de recolección de datos: la observación participante, la entrevista en profundidad, los grupos de discusión y el análisis de documentos. Se utilizó un cuestionario para recopilar información sociodemográfica y económica de la población de estudio, y determinar los grados socioeconómicos, de acuerdo con la metodología descrita por Madrigal. (1997)

El alumnado de tercero y cuarto año que conformó parte de la población de estudio, fue clasificado con obesidad, de acuerdo con los criterios emitidos por la Organización Mundial de la Salud (WHO, 1995) y la Asociación Americana de Pediatría (Dietz, Robinson, 1998; Williams, Gulli, Deckelbaum, 2001). Las niñas y los niños que resultaron con obesidad, se constituyeron en las principales fuentes de información de esta investigación.

La población estudiantil de segundo a sexto año $(n=460)$, también fue evaluada con los criterios mencionados y clasificada según el estado nutricional en: normal, con sobrepeso o con obesidad. Ello con el propósito de explorar las creencias de los niños y las niñas, según edad (8, 9, 10, 11 y 12 años) y estado nutricional, en relación con las niñas obesas y los niños obesos. Para explorar estas creencias se aplicaron dos instrumentos de frases incompletas $\dot{i}$...las niñas gordas son...? y i...los niños gordos son...? Las creencias recolectadas mediante este instrumento se clasificaron en tres categorías: neutrales, favorables o positivas y desfavorables o negativas.

Cabe mencionar que estos instrumentos no se aplicaron en la población estudiantil de primer año, porque se consideró que no era fuente de información adecuada, por su iniciación en los procesos de lectura y escritura.

Estrategias para el análisis de los datos y la construcción de significados: Para el análisis de datos cualitativos se utilizó el programa ATLAS.ti, Versión WIN: 5.0.66, al cual se asignaron 136 y el programa SPSS para Windows, Versión 10.0 se usó para realizar algunos cálculos estadísticos de tipo descriptivos. Estos programas fueron concebidos como valiosos recursos que facilitaron la organización y la clasificación de la información, así como su tratamiento, con el propósito de extraer los significados más relevantes sobre la obesidad.

El modelo de análisis de datos cualitativos, propuesto por Miles y Huberman (1994), citado por Rodríguez (1996) fue otra estrategia que se utilizó para analizar los datos. El seguimiento de este modelo implicó realizar varias tareas que se interrelacionaron entre sí, a saber: la recogida de datos, la disposición de los datos, la reducción de los datos y la extracción o verificación de las conclusiones.

Como estrategia de validación de la información; la recogida de los datos se realizó escalonadamente (Cea, 1998, p. 53); se inició con los procesos de observación y paulatinamente se aplicaron las otras técnicas e instrumentos descritos, y posteriormente se llevó a cabo el proceso de triangulación de los datos. Para conocer la fiabilidad de la calidad científica de la información, se calculó "el porcentaje de acuerdo" -entre la autora y dos asesoras del Comité de tesis-. La información recabada es válida y tiene una fiabilidad del 97,0\%.

\section{Resultados}

\subsection{Características sociodemográficas y socioeconómicas de la población en estudio}

La edad promedio de los niños obesos y de las niñas obesas fue de 10 años, con un 
rango de 8,9 años y 11,2 años. La edad promedio de sus progenitores fue de 40,9 años con un rango de 35,1 años y 45,8 años. El $31,3 \%$ había completado la educación primaria; $18,7 \%$ no terminó la secundaria y $12,5 \%$ sí. Y únicamente dos madres (12,5\%) habían terminado una carrera universitaria.

La mitad de los progenitores se dedicaba a realizar los oficios domésticos de su hogar y atender algún negocio propio. Una cuarta parte de las madres era ama de casa y la otra cuarta parte laboraba en alguna empresa pública o privada, ejerciendo funciones de docencia, administración, enfermería o de mesera en una soda o de dependiente en un bazar.

La mayoría de estas familias participantes procedía de zonas urbanas de las provincias de San José y Heredia. El $62,5 \%$ mostró una condición socioeconómica media; $25,0 \%$ baja y $12,5 \%$ alta. El $43,8 \%$ de las familias tenía una estructura nuclear; $31,2 \%$ extendida y $25 \%$ matriarcal.

\subsection{Creencias acerca de la salud, el sobrepeso y la obesidad}

De acuerdo con la mayoría de las niñas obesas (82\%) y de la totalidad de los niños obesos (100\%), ellas y ellos creen que "salud" es la ausencia de "dolor físico" o simplemente estar bien, alimentarse bien o tener una buena condición física, y que tanto la obesidad como el sobrepeso no son enfermedades. Probablemente, ésto lo creen así porque el "dolor físico" no es un síntoma común que haya manifestado esta población.

La mayoría de los niños obesos (83\%) y sólo $45 \%$ de las niñas obesas creen que la diferencia entre la obesidad y el sobrepeso estriba en el aumento de peso corporal que se da en la primera condición respecto a la segunda. Algunas expresiones son:

\footnotetext{
"Salud, no sé, puede ser que estar sin dolor de cabeza o así sin un dolor, sentirse bien, sin que nada le duela. (Niña de tercer año).

"Salud es estar saludable, estar en una buena condición física y alimentarse bien. Sobrepeso es tener
}

mucho peso y obesidad es tener más, las personas que son gordas tienen eso... son obesos.”. (Niña de cuarto año).

Asimismo, más de la mitad de las niñas obesas y sólo un niño obeso creen que entre la obesidad y el sobrepeso no hay ninguna diferencia.

Por otro lado, la mayoría de las madres $(66,6 \%)$ cree que "salud" es sinónimo de bienestar o de un estado dónde debe haber equilibrio o balance entre lo físico y lo metal. También, creen de manera similar a sus hijos obesos e hijas obesas (casos del estudio) que, salud es la ausencia de "dolor físico", alimentarse bien y tener una buena condición física. No obstante, algunas madres contemplan otros aspectos que le imprimen integralidad a la creencia de lo que es para ellas "salud".

En contraste con lo que cree la minoría de los niños obesos y de las niñas obesas, la mayoría de las madres $(73,3 \%)$ cree que la obesidad es una enfermedad o un problema de salud, pero hasta en la adolescencia y la adultez y no en los niños ni en las niñas que presentan actualmente esta condición.

Además, creen que la obesidad es una característica física "temporal" que llega a desaparecer con el paso del tiempo y que si ésta persistiera, podría constituirse durante la adolescencia en un problema más de índole "estético" y de "socialización" que de salud física, especialmente en las niñas, y de agotamiento físico (cansancio, incomodidad, movilidad limitada) en los niños y las niñas; principalmente en los primeros:

\footnotetext{
"Diay, salud es estar bien de todo, alimentarse bien para tener buena salud. Yo pienso que obesidad es una cosa y sobrepeso es otra ¿o es lo mismo?... obesidad es idiay, el chiquito que es muy gordito desde chiquitito, me imagino que si no se cuida, idiay le da a uno miedo... por la salud primero y por vanidad digo yo, que la persona idiay se acompleja mucho, verdad, una persona que es muy gordita o que en la escuela o en el colegio o quizás más grandes, las vayan a estar molestando de que es gorda, que es gordo. Aunque chiquitos bebés que nacen así gorditos verdad, cuando entran a la adolescencia se hacen altos y la gordura se les va como emparejando hasta que ya no... Aunque
} 
sino se cuidan sí puede a uno le da miedo que se sigan haciendo más y más gordos; por lo que le dije verdad, por la salud y la vanidad también. Obesidad es estar muy, muy, muy gordito. Y sobrepeso, diay estar un poquito más de lo, diay la palabra lo dice, estar un poquito más de lo que uno tiene que estar digamos de peso y en eso va la mala alimentación y falta de ejercicio. ..Quizás no ahorita, las consecuencias vienen después. Cuando esté uno más, bueno probablemente en un futuro no muy lejano verdad, cuando ya esté que se yo, llegue a la adolescencia que se ponga coqueta o así, o ya llegue a la edad un poquito más maduro y es donde uno empieza a sufrir las consecuencias". (Madre de una niña obesa de cuarto año).

De manera similar a los niños obesos y a las niñas obesas, la mayoría de las madres $(73,3 \%)$ cree que la diferencia entre la obesidad y el sobrepeso está en el aumento de peso corporal que se da en la primera condición con respecto de la segunda.

A su vez, la cuarta parte de las madres $(26,6 \%)$ creen que la obesidad no sólo será un problema de salud en el futuro sino también en la actualidad para los niños y las niñas que tienen esta condición, porque disminuye tanto la aceptación de su imagen corporal (por estética, vanidad) como la habilidad para moverse y porque creen que la obesidad desencadena otro tipo de enfermedades crónicas, tales como la diabetes, la hipertensión y las dislipidemias:

\footnotetext{
"Yo creo que la obesidad puede afectar la salud de los niños porque empiezan a padecer de un montón de cosas, incluso de colesterol y de diabetes y de todo a pequeñas edades a veces asociadas a ese tipo de problemas. No sé si existen niños que padezcan de la presión, pero me imagino que sí, de la presión por problemas de obesidad; y hasta de diabetes pueden llegar a tener. Yo creo que la obesidad los puede afectar más adelante; no solamente la parte física, sino también la parte emocional, verdad. Toda esa serie de conflictos de personalidad y de baja autoestima se empiezan a tener porque son rechazados, porque si es gordo, porque los ofenden, porque los humillan y todo ese tipo de cosas que se pueden generar, verdad (...)". (Madre de una niña obesa de cuarto año).

"Yo creo que la obesidad afecta a los niños desde pequeños; ya pueden venir padeciendo de colesterol y mucho niño ya comienza con diabetes y con problemas para moverse". (Madre de una niña obesa de cuarto año).
}

\subsection{La obesidad: es una expresión física}

Las niñas obesas y niños obesos creen que la obesidad es una forma o tipo de cuerpo que algunas personas pueden o no tener; es una característica física que la relacionan con el peso corporal y las dimensiones de su contextura física. Esta creencia podría estar respondiendo a que la mayoría no cree que la obesidad sea una enfermedad pero sí una expresión fenotípica. De ahí que estos niños y estas niñas le asignaron varios sinónimos a la persona que es obesa; tales como: gorda o gordita, pesada o pesadita, redondita, ancha y gruesa o gruesita, entre otros.

Asimismo, al creer ellos y ellas que la obesidad es una característica física; le otorgan a esta condición cierto grado de relatividad o de escala. Es decir, para ellos y ellas, una persona puede ser obesa, pero sólo un poco. También, se puede observar que no es una condición deseable, por lo que la mayoría de las niñas que expresaron que sí estaban gorditas agregaron que sólo un poquito e incluso un niño de cuarto año lo negó.

Por ello, cabe destacar que las niñas obesas y los niños obesos califican a sus pares que tienen obesidad como pesados o gordos; pero cuando se autocalifican, dicen que están algo pesados o un poquito gordos o gordas:

\footnotetext{
"Las niñas obesas son muy gordas y pesan mucho y los niños obesos son también muy gordos y pesados. Si alguien me preguntara si yo soy un niño obeso, yo le diría que sólo estoy un poco pasado del peso que tengo que tener.". (Niño de cuarto año).

"Mi abuelita y yo, eh... mi abuelito, mi mamá y yo estamos como unos embarazados. Las niñas obesas son gordas, cómo es... sudan y comen mucho y los niños obesos también igual, son gordos de peso y comen mucho. Si alguien me preguntara si yo soy una niña obesa, yo le diría que un poco, sí. La gente ancha es así porque come y come mucho.”. (Niña de tercer año).

"En mi casa todos son gorditos... porque son redonditos, así son ellos, son redondos. En las fotos hay gente que es familia de ellos y bueno mía también (risas) que son así redonditos". (Niña de cuarto año).
} 
Por otra parte, al igual que sus hijas e hijos (casos del estudio), todos los progenitores creen que la obesidad es una expresión física o forma corporal que algunas personas o toda una familia pueden o no tener, y relacionaron la obesidad con el peso corporal y las dimensiones de la contextura física de la persona, señalándola como: gorda o gordita, muy pasada de peso, ancha, gruesa o gruesita, grandota, panzoncita, rellenita, regordita, pesadita, muy gorda, con contextura grande, con esqueleto grande y ancho, con espalda ancha, de huesos anchos, entre otros.

Asimismo, todos los progenitores, excepto dos madres, creen que una persona puede ser obesa, pero sólo un poquito, ya sea porque consideran que tiene un poco más de peso o porque está un poquito pasada e incluso una madre cree que su hijo obeso está entre lo normal de los gorditos.

Es decir, los progenitores no creen que una persona sea obesa en sentido categórico o radical. Esta condición de "relatividad" que le otorgan a la obesidad podría interpretarse como una negación a la obesidad en sentido absoluto. Ello sugiere que en las familias estudiadas existe la creencia de que la obesidad no es una condición deseable:

"Bueno, no todos pero de parte de mi familia casi todas mis tías son gruesas, casi todas son gruesas, que por cierto ella (se refiere a su hija "B") lleva un cuerpo como de mis tías verdad, un cuerpo grueso. Lo que pasa es que ella tiene unas tías que están muy pasadas vea, de peso ya, pero si casi todas son gruesas. Después de parte de mi esposa casi todas las tías también son gruesas verdad. Y la abuela de ella, la mamá de mi esposa es gruesa, las hermanas de ella también son gruesas, entonces vienen, como que viene una descendencia toda gruesa verdad. ...pero diay se mantiene ahí". (Padre de una niña obesa de cuarto año).

"E”, no pero, pues... si sé que está obeso porque, sé que está un poquito pasado a lo que él debería de estar, pero tampoco lo considero como un niño que esté regordito... regordito... no; entre lo normal de los gorditos, digamos. Yo creo que las niñas gordas son las que están bien rellenitas... las que están bien pesaditas... y los niños gordos igual... los que están más... o bien pesaditos, que se ven muy gorditos, muy rellenitos.”. (Madre un niño obeso de cuarto año).

\subsection{Las creencias sobre la obesidad y sus implicaciones psicosociales}

En relación con que la obesidad representa una apariencia física o condición no deseable, cabe destacar las creencias de algunos niños obesos, quienes utilizaron adjetivos despectivos para describir a las niñas obesas:

"Las niñas obesas son gordas, un poco feas y que no se alimentan bien (...).”. (Niño de tercer año).

"Las niñas obesas son algo... como le digo, no son muy atractivas; como que queeeeee modelos no (...).”. (Niño de cuarto año).

De igual manera, las niñas obesas expresaron sus sentimientos en relación con su condición, haciendo alusión a experiencias burlescas que han vivido en la propia escuela. En sus relatos se puede constatar que existe en ellas una actitud de defensa de frente a las opiniones de los niños obesos:

"Si alguien me preguntara si yo soy una niña obesa, yo le diría que sí pero que no me moleste más. O así que me dicen cachetona o que me hacen como chancho.”. (Niña de cuarto año).

"Las niñas obesas son gordas también y no les gusta que les digan cosas, así que las quieran humillar. Si alguien me preguntara que si yo soy una niña obesa, yo le contestaría que no me ofenda. Es que yo me sé defender". (Niña de cuarto año).

En relación con "el complete" que hicieron por escrito, las 235 niñas y los 225 niños, de segundo a sexto año matriculados en la escuela, caso de estudio, se puede afirmar que se confirmaron los patrones de creencias descritos. El confronto de los datos obtenidos con el instrumento de "freses incompletas" y las entrevistas realizadas, permitió aplicar el círculo hermenéutico (que va de lo general a lo particular y viceversa), contextualizar la información brindada por los niños y las niñas y tener 
una perspectiva más crítica sobre el fenómeno en estudio.

Los datos del cuadro 1 reflejan que las opiniones de los niños y de las niñas son más favorables para los niños obesos que para las niñas obesas. El $40,4 \%$ de los niños y $23,4 \%$ de las niñas opinan favorablemente sobre los niños obesos, mientras que sólo $10,2 \%$ de los niños y $20,4 \%$ de las niñas opinan favorablemente sobre las niñas obesas; existiendo diferencias estadísticamente significativas entre las opiniones de los niños (40,4\% vrs $10,2 \%$, $Z=7.260, p<0,000)$.

Cuadro 1

Creencias que tiene la población estudiantil de segundo a sexto año de la Escuela en estudio, sobre las niñas obesas y los niños obesos, según sexo y año

\begin{tabular}{|c|c|c|c|c|c|c|}
\hline \multirow[b]{3}{*}{ Año } & \multicolumn{6}{|c|}{ "Las niñas gordas son..." } \\
\hline & \multicolumn{3}{|c|}{ Niños $(n=225)(\%)$} & \multicolumn{3}{|c|}{ Niñas (n=235) (\%) } \\
\hline & $\mathrm{n}(\mathrm{a})$ & $+(b)$ & $-(c)$ & $\mathrm{n}(\mathrm{a})$ & $+(b)$ & $-(c)$ \\
\hline $2^{\circ}$ & 28,6 & 21,4 & 50,0 & 56,3 & 25,0 & 18,8 \\
\hline $3^{\circ}$ & 35,7 & 4,8 & 59,5 & 65,1 & 9,3 & 25,6 \\
\hline $4^{\mathrm{o}}$ & 27,7 & 10,6 & 61,7 & 36,5 & 21,2 & 42,3 \\
\hline $5^{\mathrm{o}}$ & 20,0 & 11,1 & 68,9 & 37,8 & 26,7 & 35,6 \\
\hline $6^{\circ}$ & 16,3 & 4,1 & 79,6 & 36,2 & 19,1 & 44,7 \\
\hline Total & 25,3 & 10,2 & 64,4 & 46,0 & 20,4 & 33,6 \\
\hline
\end{tabular}

\begin{tabular}{rrrrrr}
\multicolumn{5}{c}{ "Los niños gordos son...” } \\
\hline \multicolumn{3}{c}{ Niños (n=225) } & $(\%)$ & \multicolumn{3}{c}{ Niñas (n=235) $(\%)$} \\
n (a) & + (b) & \multicolumn{1}{c}{ - (c) } & n (a) & + (b) & - (c) \\
\hline 28,6 & 71,4 & 0,0 & 25,0 & 31,3 & 43,8 \\
35,7 & 50,0 & 14,3 & 34,9 & 16,3 & 48,8 \\
57,4 & 29,8 & 12,8 & 46,2 & 26,9 & 26,9 \\
60,0 & 31,1 & 8,9 & 42,2 & 24,4 & 33,3 \\
61,2 & 24,5 & 14,3 & 40,4 & 17,0 & 42,6 \\
\hline 49,3 & 40,4 & 10,2 & 37,9 & 23,4 & 38,7 \\
\hline
\end{tabular}

(a) = opinión neutral

(b) = opinión positiva o favorable

(c) = opinión negativa

Cabe destacar que $64,4 \%$ de los niños tiene una opinión negativa sobre las niñas obesas, mientras que, sólo $10,2 \%$ de ellos opinan negativamente sobre los niños obesos (Cuadro 1); siendo estas diferencias estadísticamente significativas $(64,4 \%$ vrs $10,2 \%, Z=11.790, p<0,000)$. En el caso de las niñas esta diferencia fue pequeña (menor al 6\%) y no fue estadísticamente significativa; $33,6 \%$ de ellas tuvo una opinión negativa sobre las niñas obesas y $38,7 \%$ sobre los niños obesos (Cuadro 1).

Además, los datos muestran una tendencia acentuada hacia una creencia que refleja desprecio a la persona obesa, conforme aumenta la edad y especialmente entre los niños respecto a las niñas obesas. Por ejemplo, $50 \%$ de los niños de segundo año opina en forman negativa sobre las niñas obesas y este porcentaje aumenta hasta $79,6 \%$ entre los niños de sexto año.
Es decir, las opiniones negativas hacia las niñas obesas entre los niños de sexto año se acrecientan en una tercera parte respecto a los niños que están en segundo año.

Por otro lado, los datos según estado nutricional (Cuadro 2) muestran que las niñas con estado nutricional normal son las que mayoritariamente opinan en forma negativa sobre las niñas obesas. Por ejemplo, 53,2\% de las niñas con estado nutricional normal opina en forma negativa de las niñas obesas, mientras que sólo opinan así, $13,5 \%$ de las niñas obesas y 7,6\% de las niñas con sobrepeso. Este comportamiento de opinión negativa acerca de sus pares del mismo sexo y que presentan obesidad, se mantiene entre los niños, $15,8 \%$ de los niños con estado nutricional normal opina en forma negativa sobre los niños obesos y sólo $6,8 \%$ de los niños con obesidad y $2,6 \%$ con sobrepeso opinan así. 
Cuadro 2

Creencias que tiene la población estudiantil de segundo a sexto año de la Escuela en estudio, sobre las niñas obesas y los niños obesos, según sexo y estado nutricional

\begin{tabular}{|c|c|c|c|c|c|c|c|c|c|c|c|c|}
\hline \multirow{3}{*}{$\begin{array}{l}\text { Estado } \\
\text { nutricional }\end{array}$} & \multicolumn{6}{|c|}{ Creencias sobre "las niñas gordas son..." } & \multicolumn{6}{|c|}{ Creencias sobre "los niños gordos son..." } \\
\hline & \multicolumn{3}{|c|}{ Niños $(n=225)(\%)$} & \multicolumn{3}{|c|}{ Niñas $(n=235)(\%)$} & \multicolumn{3}{|c|}{ Niños $(n=225)(\%)$} & \multicolumn{3}{|c|}{ Niñas $(n=235)(\%)$} \\
\hline & n (a) & $+(b)$ & $-(\mathrm{c})$ & $\mathrm{n}(\mathrm{a})$ & $+(b)$ & $-(c)$ & $\mathrm{n}(\mathrm{a})$ & $+(\mathrm{b})$ & $-(c)$ & n (a) & $+(b)$ & $-(\mathrm{c})$ \\
\hline Normal & 25,8 & 16,7 & 57,5 & 24,2 & 23,5 & 52,3 & 55,0 & 29,2 & 15,8 & 47,0 & 31,1 & 22,0 \\
\hline Sobrepeso & 28,4 & 4,1 & 67,6 & 75,8 & 16,7 & 7,6 & 43,2 & 54,1 & 2,7 & 24,2 & 16,7 & 59,1 \\
\hline Obesidad & 13,8 & 0,0 & 86,2 & 70,3 & 16,2 & 13,5 & 41,4 & 51,7 & 6,9 & 29,7 & 8,1 & 62,2 \\
\hline Total & 25,3 & 10,2 & 64,4 & 46,0 & 20,4 & 33,6 & 49,3 & 40,4 & 10,2 & 37,9 & 23,4 & 38,7 \\
\hline
\end{tabular}

(a) = opinión neutral

(b) = opinión positiva o favorable

(c) = opinión negativa

No obstante, cuando se refirieron a sus pares con obesidad y que eran de diferente sexo al de ellas o al de ellos; las niñas y los niños con sobrepeso u obesidad mostraron que sus sistemas de creencias acerca de las niñas obesas y los niños obesos, respectivamente, consistían en representaciones sociales cargadas de ideas preconcebidas relacionadas con la fealdad y la torpeza para moverse, entre otros prejuicios. Por ejemplo, 59,1\% de las niñas con sobrepeso y $62,2 \%$ de las que presentaron obesidad opinaron en forma negativa sobre los niños obesos. En el caso de los niños, 67,6\% que presentó sobrepeso y $86,2 \%$ obesidad, opinaron de forma negativa acerca de las niñas obesas. Cabe destacar que en estas opiniones, los niños obesos aventajaron a las niñas obesas en más de un $20 \%$ de opiniones negativas y esta diferencia resultó estadísticamente significativa $(86,2 \%$ vrs $62,2 \%, Z=5.755, p<0,000)$.

Las niñas y los niños brindaron una variedad de respuestas que reflejan sus creencias; algunas sólo se refieren a cómo creen ellas y ellos que son las niñas obesas y los niños obesos, principalmente, desde el punto vista físico (creencias neutrales): anchas, altas, gordas, pequeñas, bajos, gordos, entre otros adjetivos que no son ofensivos ni halagadores.
Otras respuestas muestran que las niñas y los niños creen que sus pares que presentan obesidad son personas con cualidades deseables, tales como, buenas, inteligentes, amigables, simpáticas e incluso con belleza física (creencias positivas). Asimismo, se puede observar una serie de respuestas que expresan que las niñas y los niños creen que sus pares con obesidad son desagradables y hasta repulsivos a la vista y al olfato.

Cabe destacar que los adjetivos más frecuentemente usados por los niños y las niñas, para describir a las niñas obesas fueron: feas, asquerosas, horripilantes, enojonas, ridículas, amargadas y chanchas. Y para describir a los niños obesos fueron: feos, muy cansados al correr, lentos corriendo, groseros, huelen mal, burlones y perezosos. Estos sistemas de creencias están cargados de estereotipos que obedecen en parte al ideal social de cuerpo esbelto, pues éste es considerado como sinónimo de belleza, éxito y actividad.

Por otra parte, de acuerdo con los discursos de todos los progenitores, ellos creen que la obesidad representa un problema estético y social para las personas obesas. Asimismo, creen que este problema "estético" no está repercutiendo en algunas niñas y niños, porque no han entrado a la adolescencia. 
Esta creencia promueve en los progenitores (sobre todo en las madres) aconsejar a sus hijos e hijas, a no comer en exceso y a realizar ejercicios, con el objetivo de que puedan sentirse bien con su figura corporal y logren socializar con sus pares. Incluso, creen que la obesidad podría ser una barrera importante para que sus hijos e hijas inicien un noviazgo:

“...porque a ella (se refiere a su hija "B”) le dicen que está gorda y a ella le da igual verdad. Entonces pienso que vive su vida, también le voy a decir algo, diay ella es una niña, pero cuando ella llegue a una edad donde le gusten los muchachillos, verdad, y es que usted sabe que es verdad, yo lo viví, yo estuve en el cole y yo todo eso lo estudié y cuando uno ve a una muchacha muy gruesa, bueno a mí nunca me gustaron las gordas, verdad, honestamente a mí me gustaba la chamaca más o menos, flaquita y bonita verdad, pero ya una gorda como que a uno no le llama la atención, verdad. ...pero yo siento que ella, por el momento, pues, está bien, se siente bien, habría que verla ya cuando entre a una etapa de adolescente, verdad, donde ya a ella le llame la atención un güililla y cosas así. Entonces ya ahí yo pienso que ellas también van buscando como para verse bonitas verdad. ... mi esposa, mi hija la otra, la que es un poquito mayor que ella, y ella le dice: "B" cuídese porque se va a hacer muy gorda, y vea que las mujeres gordas son muy feas verdad, y eso le dicen. Y después ya usted va a entrar en una etapa en que le gustan los chiquillos y los chiquillos la ven así. Y entonces ella le hace ver todo así verdad, y entonces mi esposa sí le ha hablado mucho, vea "B" tiene que cuidarse, tiene que dejar de comer tal cosa para que no engorde tanto, y todo eso, lo que pasa es que ella es una niña verdad. (...) ella nada más me da quejas de la hermana que a veces le dice que está muy gorda y que la hermana sólo decirle gorda y cosas así verdad". (Padre de una niña obesa de cuarto año).

\subsection{Creencias sobre las prácticas de alimentación y actividad física}

Las niñas obesas y los niños obesos y sus progenitores creen que la obesidad es un estado que puede revertirse mediante el control de la ingesta alimentaria (haciendo dieta o estando encima de la persona para que no coma). Se considera que esta creencia, podría estar respondiendo a que la totalidad de los progenitores y de las niñas obesas y de los niños obesos creen que la sobre alimentación (comer en exceso o mucho) es la causa o una de las causas de la obesidad, tanto en las personas adultas como en los niños y las niñas.

También, creen que el control de la ingesta es necesario porque sino se deja de cumplir la dieta, ya sea por olvido o por falta de motivación, y que la sobre-ingesta es atribuible a la presencia de ansiedad:

"Yo pienso que algunas personas adultas son gordas o gordos porque comen mucho. Y las niñas y los niños que son así gordos, también es porque comen mucho. ...estamos gordos, yo, mis hermanas y mi mamá; este,...por tener las bebés y que se le hicieron estrías y todo (se refiere a su hermana) y mi mamá por tener tantos hijos, y yo por comer demasiado. Yo paso come y come, es que yo tengo ansiedad y mi mamá me daba pastillas para eso pero las traían de Nicaragua y eran muy caras. ...pero ya se me quitó eso, antes pasaba come y come cada diez minutos y ahora ya no tanto". (Niño obeso de cuarto año).

"En mi casa nosotros todos somos gruesos. Creo que estamos así... bueno yo me divorcié hace, lo del divorcio y toda la cosa, por tensión... porque en realidad yo como bien pero he tenido unos ataques de ansiedad terribles y yo me imagino que en realidad parte del problema de "J" (se refiere a su hijo J") fue ese también porque él hasta que estuvo en la escuela fue que empezó a engordar". (Madre de un niño obeso de tercer año).

Otras creencias observadas fueron que: -el consumo de frutas, verduras, vegetales y agua, adelgaza; - el consumir vegetales y frutas equivale a tener una alimentación nutritiva y balanceada; - una estrategia efectiva para adelgazar es "dejar de comer entre comidas o en la cena" o, comer menos en ese tiempo de comida y evitar las frituras, las golosinas, las harinas y en general la comida chatarra:

"Comer bien, equilibrado, cosas nutritivas; no comer entre comidas, tomar agua y hacer ejercicios. Para estar saludable, yo me mido, no como mucho ni tan poquito. Trato de comer frutas y ensaladas. "A" (se refiere a su hijo "A"), yo trato y él trata porque si le sirvo se come la ensalada y las frutas. Tomamos agua, tratamos de no comer entre comidas y hacemos ejercicio, él jugando y yo con los oficios y a veces cuando camino hago más ejercicios. (...) primero, creo que la soda puede cambiar lo que vende. En el comedor 
creo que deben dar más frutas y ensaladas". (Madre de un niño obeso de cuarto año).

"Mi mamá dice que es muy bueno que después de que ella hace digamos arroz, frijoles, carne que después de eso comer ensalada porque la ensalada mata la grasa, o sea quema la grasa que uno comió. Los que están a dieta en mi casa son mi hermana; yo a veces y mi mamá (...) mi hermana trata de no comer en la noche por más rica la comida (...). Ejercicios, a veces empiezo a bailar con mi hermana, o jugamos bola. Ella (se refiere a su hermana) elimina mucha grasa y no come mucho en la noche; y yo, no como mucho en la noche, como poquito y digamos si hizo (se refiere a su madre) ensalada en la mañana, yo le digo que me la guarde para comérmela en la noche. Yo le quitaría (se refiere a la soda de su escuela) la comida chatarra como la pizza, las empanadas son con mucha grasa”. (Niña obesa de cuarto año).

\subsection{El sedentarismo, causa de la obesidad}

Se encontró que dos niñas obesas $(18,2 \%)$, un niño obeso $(16,7 \%)$ y seis de sus progenitores $(37,5 \%)$ creen que el sedentarismo es una de las causas de la obesidad, tanto en las personas adultas como en los niños y las niñas. Además, creen que el sedentarismo es a la vez una consecuencia de la obesidad y que las niñas obesas y los niños obesos son personas perezosas, característica que conciben como una barrera importante para la realización de alguna actividad física:

"Están gordos, digamos que mis hermanos (...) no hacen nada de ejercicio y solo comen, si hicieran por lo menos un poquito de ejercicio como que se contrarresta verdad, pero si no (...) este, la obesidad desde ahorita afecta en todo; digamos un niño obeso es un niño más perezoso para caminar, para hacer ejercicio, para todo". (Madre de una niña obesa de cuarto año).

\section{Discusión}

De acuerdo con las creencias de las niñas y los niños, "la salud" es un estado radical que se puede tener o no tener; por lo tanto, "la salud" no tiene cabida como un proceso dinámico. Mientras que las creencias de sus progenitores acerca de la salud son "más integrales" y complejas que las de las niñas y los niños. Esto es de esperar porque la mayoría de los progenitores tienen más años de estudio y por supuesto que mayor cantidad de experiencias de vida que sus hijas e hijos. En el diario compartir con los semejantes, los seres humanos tienen más probabilidades de valerse de muchos más elementos de diversa índole (sociocultural, cognitivo y afectivo) para construir y modificar sus creencias.

Considerando el referente de MaxNeff, y otros (1986) (Cuadro 3) que reúne categorías que oscilan desde una representación simple de la salud hasta una representación compleja e integral de la misma; las creencias acerca de la salud de la mayoría de las niñas obesas y de los niños obesos están relacionadas con la categoría menos compleja, es decir con la primer categoría correspondiente a la "ausencia de enfermedad", mientras que las creencias acerca de la salud de la mayor parte de sus progenitores abarcan esa categoría y las categorías relacionadas con el "énfasis en salud física" y el "énfasis en la salud mental y emocional".

Las creencias representan visiones de mundo, historias de vida, interacciones sociales y comunicaciones verbales ricas en lenguaje, modismos y costarriqueñismos, y son utilizadas por las personas como instrumentos de lectura e interpretación para seleccionar y organizar las informaciones que reciben así como para establecer relaciones entre ellas. En consecuencia, las niñas y los niños se han forjado, en su seno familiar e interactuando con sus figuras referenciales y otras personas, una representación de lo que es salud, sobrepeso y obesidad -entre otras nociones-.

Así también, se han hecho una representación a lo largo de los años, de ellas mismas y de ellos mismos como personas obesas y han relacionado a la obesidad con varios aspectos de diversa índole; de tal manera que van produciendo distintos significados para la obesidad. Entre éstos 


\section{Cuadro 3}

Indicadores para estudiar las creencias acerca de la salud y la obesidad en las niñas obesas, los niños obesos y sus progenitores

\begin{tabular}{|c|c|}
\hline Categorías & Indicadores \\
\hline Ausencia de enfermedad & No estar enfermo, no visitar al médico \\
\hline Énfasis en salud física & $\begin{array}{l}\text { Alimentación } \\
\text { Ejercicios } \\
\text { Abrigo } \\
\text { Higiene hogar } \\
\text { Higiene personal } \\
\text { Vacunación } \\
\text { Medicinas } \\
\text { Visita al médico } \\
\text { Instituciones de salud }\end{array}$ \\
\hline Énfasis en salud ambiental & $\begin{array}{l}\text { Aire puro } \\
\text { Ambiente sano } \\
\text { Agua potable } \\
\text { Ausencia de desechos } \\
\text { Sin contaminación } \\
\text { Zonas verdes } \\
\end{array}$ \\
\hline Énfasis en la salud mental y emocional & $\begin{array}{l}\text { Descanso } \\
\text { Equilibrio/orden } \\
\text { Seguridad } \\
\text { Derechos } \\
\text { Amistades } \\
\text { Autoestima } \\
\text { Respeto } \\
\text { Juegos y recreación } \\
\text { Tranquilidad } \\
\text { Sin adicciones } \\
\text { Solidaridad } \\
\text { Cooperación } \\
\end{array}$ \\
\hline Énfasis en salud espiritual & Ser superior, Dios \\
\hline
\end{tabular}

que la obesidad representa una apariencia física desagradable o no deseable.

Respecto a lo que la mayoría de las niñas obesas y los niños obesos y sus progenitores dicen que hacen para estar saludables, se puede constatar que sus creencias preceden a sus prácticas, pero éstas a su vez refuerzan sus creencias acerca de la obesidad y de cómo controlarla. Tanto las creencias como sus prácticas se pueden ubicar en la segunda categoría "salud física" (indicadores sobre alimentación y ejercicios, cuadro 3) y están relacionadas con las creencias que tienen ambos (progenitoreshijas e hijos) acerca de que la sobre-ingesta $\mathrm{y}$ el sedentarismo son las principales causas de la obesidad. Por ejemplo, tomar agua para controlar la ansiedad de comer, disminuir o eliminar la ingesta en la cena y hacer ejercicios en una máquina.

Estas creencias acerca de las causas de la obesidad que tienen progenitores, niñas y niños, se podrían explicar en gran parte por la información brindada por los medios de comunicación masiva que transmiten tanto contenidos científicos como de tipo simbólico que le rinden culto al cuerpo esbelto. Es decir, el caldo de cultivo que alimenta a las creencias no se circunscribe al seno familiar o doméstico u hogareño, sino que se impregna de las creencias de la sociedad, las cuales se transmiten de 
generación en generación y se recrean en las múltiples interacciones sociales.

De acuerdo con la influencia social en la construcción de las creencias; en la literatura científica sobre las prácticas que realizan las niñas y los niños para controlar el peso corporal, se afirma que están relacionadas con tres factores principales: a) reciben crítica y presión para tener una figura atractiva por parte de sus progenitores y pares, b) no son felices en el seno familiar y c) están expuestos a los medios de comunicación que divulgan diferentes métodos para el control del peso corporal (Field, Cheung, Wolf, Herzog, Gortmaker, Colditz, 1999; Hill, Franklin, 1998; McCabe, Ricciadelli, 2001; Muris, Meesters, Van de Blom, Mayer, 2005; Presnell, Bearman, Stice, 2004; Zalilah, Anida, Merlin, 2003).

De hecho en este estudio etnográfico, las niñas y los niños manifiestan sus creencias acerca de la obesidad enmarcándose en aspectos o experiencias de carácter emocional. Explícitamente, el sobrepeso y la obesidad no son considerados por ellas y ellos como patologías. Y a diferencia de sus creencias acerca de "la salud" como un estado que se posee o no, sus creencias sobre la obesidad sí contemplan que una persona pueda ser un poquito obesa, pues creen que la obesidad es una característica física referida a las dimensiones de contextura o peso corporales. Asimismo, algunas niñas y algunos niños relacionan a la obesidad con la presencia de sudor y otros aspectos desagradables; hasta tal punto que creen que sus pares son repulsivos a la vista y al olfato. Estas creencias se pueden ubicar en la segunda categoría "salud física" (indicador sobre higiene personal, Cuadro 1).

Por otro lado, es importante destacar que las creencias de los progenitores acerca de la obesidad son diferentes de acuerdo con las etapas de la vida del ser humano. Es decir, sus creencias sobre la obesidad son transversales o cronológicas; los progenitores sitúan al ser humano en el transcurrir del tiempo y dependiendo de éste, creen que la obesidad es o no un problema de salud con consecuencias físicas, motoras o estéticas. Por ejemplo, la mayor parte no cree que la obesidad sea una enfermedad o un problema de salud durante la niñez ni en la vejez. Incluso las adultas mayores y los adultos mayores y su relación con la obesidad están ausentes en las creencias de los progenitores. Y las adolescentes y los adolescentes son tomados en cuenta por los progenitores únicamente cuando expresan sus creencias sobre la obesidad como un problema estético.

Los resultados de esta investigación etnográfica sugieren que, las niñas obesas, los niños obesos y sus progenitores no sienten responsabilidad por el autocuidado de su salud y el control del peso corporal, a pesar de que dicen saber qué deben hacer para estar saludables. En sus discursos reclaman ayuda de alguien externo para que les oriente y sobre todo para que les "controle" o "supervise" el cumplimiento de prácticas, hábitos de alimentación y actividades física saludables.

De hecho entre las creencias y las prácticas de las niñas, los niños y sus progenitores, existen actuaciones o comportamientos acordes y también contradictorios en cuanto a lo que dicen creer que se debe hacer para prevenir la obesidad y lo que realmente dicen que hacen. Este comportamiento que tienen ambos grupos de participantes (progenitores-hijas e hijos) podría estar explicado en la teoría de Rogoff (2002) que señala que los adultos, las adultas, los niños y las niñas quedan emocionalmente atrapados en encuentros cooperativos que perpetúan en sus acciones una serie de prácticas sociales transmitidas de generación en generación, aprenden conocimientos, creencias, habilidades y valores promovidos por el grupo social al cual pertenecen.

Todo lo anterior, sugiere que en la planificación y el desarrollo de los procesos cognitivos-intelectuales-académicos acerca de alguna noción o concepto de interés para 
la educación y la salud pública (tal como la obesidad), se debe contemplar la naturaleza social, ontológica y axiológica de las creencias de esa noción o concepto. Además, que estos procesos deben ser contextualizados, considerando el seno familiar, el escenario escolar y de barrio-comunitario y la sociedad en general, pues desde antes del ingreso a la escuela se comienzan a gestar las creencias acerca de diversos conceptos o nociones y también las prácticas sociales alrededor de esas creencias.

Es decir, para lograr el cambio deseado en las creencias acerca de la obesidad y la reestructuración hacia un conocimiento cualitativamente superior sobre esta problemática, se requiere la activación de teorías previas en los aprendices y la confrontación de sus conocimientos y creencias, de acuerdo con las posiciones conflictivas, mediados por la experiencia de aprendizaje (Pozo, 1996).

Estos aspectos deben ser considerados para la conducción de los procesos de enseñanza y aprendizaje informales y formales acerca de la prevención de la obesidad, por parte de los planificadores a cargo de los programas educativos de la Educación General Básica, el personal docente y de salud, e incluso por parte de los progenitores. El éxito de estos procesos se dará en la medida en que se le facilite a la niña y al niño encontrarle sentido a una situación desafiante de aprendizaje acerca de lo que es salud y obesidad, y se promuevan espacios de reflexión crítica sobre el significado de la obesidad en la vida de las personas que la presentan y que no poseen esta condición.

Estas estrategias educativas son requeridas porque la obesidad como fenómeno educativo está afectando el desarrollo del alumnado (Hill and Silver, 1995). Ello porque entre el aprendizaje y el desarrollo existe una relación de tipo dialéctica. Para Vigotsky (1978) el aprendizaje precede al desarrollo y señala que una enseñanza adecuada es aquella que contribuye a crear zonas de desarrollo próximo; las cuales sirven de puente para hacer que el nivel potencial de desarrollo del educando o de la educanda se integre con el actual. Es un diálogo entre el niño o la niña y su futuro, entre lo que es capaz de hacer hoy y lo que será capaz de hacer mañana y no entre el niño o la niña y su pasado.

Por consiguiente, el concepto de zona de desarrollo próximo de Vigotsky cobra importancia en los procesos de enseñanza y aprendizaje sobre la prevención de la obesidad, pues las niñas y los niños que presentan esa condición la han adquirido a lo largo del tiempo, durante el cual han conformado y reforzado sus creencias acerca de la obesidad, así como hábitos de alimentación y de actividad física inadecuados.

Además, como se observó en esta investigación, la mayoría de las niñas y de los niños cree que la obesidad representa un aspecto físico desagradable y por lo tanto, no deseable. De igual manera resulta interesante la preocupación sobre la competitividad en los varones, pues algunos de los calificativos más utilizados por ellos para expresar sus creencias acerca de los niños obesos fueron muy cansados al correr, lentos corriendo y perezosos.

Todos estos aspectos de índole psicosocial fueron acotados por la mayoría de los progenitores como una preocupación importante y han sido reportados en la literatura como provocadores de baja autoestima en las niñas y los niños (Hill and Silver, 1995), lo cual les podría imposibilitar avanzar a un ritmo adecuado en sus progresos de aprendizaje y desarrollo.

Dichos estigmas que son reflejo de las creencias sobre la obesidad, no facilitan que la niña obesa y el niño obeso puedan avanzar mediante procesos colaborativos, afables y de interacción con sus pares y fundamentalmente, con independencia y autonomía, hacia un estado de salud óptimo, acorde con un entendimiento de la obesidad como problema, que los impulse a tomar la decisión de realizar acciones correctivas, pero a la vez significativas y 
con sentido para sus vidas (comprensión y actuación adecuadas ante la enfermedad que padecen).

No se puede dejar de lado considerar que el ser humano es una individualidad y la influencia de su clase social se refracta en su conciencia, mediante su experiencia personal, su carácter, sus peculiaridades y su temperamento (Solano, 1987). Por ello, dichos conflictos conceptuales y empíricos acerca de la obesidad no podrían ocurrir de manera inmediata; su continua reestructuración se realizaría gradualmente y en muchas ocasiones acompañada de dolor. En estos procesos cobra importancia la humillación y la burla que los niños obesos y las niñas obesas experimentan a lo largo de su vida estudiantil, tanto en la escuela, como en el hogar y el barrio, entre otros contextos más mediatos, por parte de las personas con las que ellos y ellas interaccionan.

Finalmente, para lograr actuaciones acordes con estilos de vida saludables y sostenibles en el tiempo, es imprescindible considerar las creencias y sentimientos de las personas obesas; es decir, la parte significativa-afectiva de la obesidad. Schumann (1999) afirma que el ser humano está rodeado de una serie de factores que lo moldean como actor pensante dentro de la sociedad y que va interiorizando los valores y las actividades que son significativas para su grupo sociocultural de pertenencia.

\section{Conclusiones y recomendaciones}

Para las niñas y los niños de esta investigación "la salud" no es un proceso, sino que representa un estado exento de síntomas físicos (dolor). Este concepto tan simplista y biologicista de la salud, sugiere que estas niñas y estos niños no han participado en procesos educativos formales (escenario escolar) ni informales (en su hogar -escenario familiar y comunitario-) donde hubiesen logrado construir un concepto holístico de la salud. Ello, a pesar de que la salud (como término genérico) para sus progenitores está relacionada no sólo con el bienestar físico, sino también con la dimensión mental y emocional de las personas.

Este estudio demuestra de manera contundente que la obesidad representa para el alumnado, más que un estado no saludable, una apariencia física desagradable o no deseable, y que tanto las niñas, los niños y los progenitores, señalan la sobre-ingesta y el sedentarismo como las principales causas de la obesidad.

En este estudio se hizo evidente que las niñas, los niños y los progenitores realizan algunas prácticas que de acuerdo con sus creencias sobre lo que engorda o causa obesidad, por lo que estos resultados sugieren que las creencias preceden a las prácticas y que a su vez, estas últimas reafirman las creencias acerca de la obesidad y de cómo controlarla.

Los sujetos participantes en este estudio, realizan las prácticas de manera intermitente, es decir, por períodos de tiempos muy cortos y no consecutivos; lo que sugiere dos situaciones: a) que sus intenciones están más centradas en perder peso para verse mejor que en perder peso para ganar salud.

Ello respalda la conclusión de que la obesidad no es considerada un problema de salud grave sino que realmente ha adquirido una representación social de apariencia física desagradable o no deseable, tanto para la niñez como para las personas adultas, pues son los progenitores (madres) los que alientan a sus hijas e hijos a realizarlas, además, de que las niñas y los niños imitan las conductas de sus progenitores (mediante el modelaje) y b) en el seno familiar, el consumo de una alimentación saludable y la ejecución de ejercicios no están siendo cultivados en las niñas y en los niños como hábitos de vida saludables.

La representación social de la obesidad quedó contextualizada con a) las experiencias emotivas que relataron los niños obesos y principalmente las niñas obesas, 
quienes acusan haber sido, con frecuencia, blanco de burla en sus hogares y sobre todo en la escuela y b) las expresiones despectivas que la mayoría del alumnado utilizó para describir a las niñas obesas y los niños obesos.

Finalmente, en este estudio se concluye que la creencia acerca de la obesidad en los progenitores es mucho más compleja que en las niñas y los niños. Los progenitores además de creer que la obesidad es una expresión externa no deseable, le van otorgando a esta condición (a la obesidad) diferentes matices, implicaciones y consecuencias de acuerdo con la etapa de vida en que se encuentre la persona que la presenta:

a) durante la infancia (cuando están "bebés") y la niñez (pre-escolar y escolar): creen que la obesidad no es un problema de salud sino una característica física "temporal", que limita las habilidades físicas de movilidad y desplazamiento

b) en la adolescencia: creen que la obesidad es un problema estético y una barrera para que puedan socializar con sus pares y experimentar el noviazgo

c) en la adultez (personas maduras pero no ancianas): creen que la obesidad es un problema de salud que lo relacionan con el desarrollo de enfermedades crónicas, tales como: la diabetes, la hipertensión arterial, las dislipidemias, y como una limitante para moverse y desplazarse.

Por lo tanto, los sectores educación y salud deben considerar en el diseño de las intervenciones y campañas de prevención de la obesidad, el equilibrio entre el uso del leguaje técnico/cientificista y el leguaje popular para mejorar la efectividad de las mismas. En este estudio, las niñas, los niños y sus progenitores utilizaron diversos sinónimos para referirse a la obesidad.
Asimismo, de forma urgente y "obligatoria", deben contrarrestar la legitimación social y la aculturación del desprecio hacia las personas obesas, especialmente hacia las niñas. Es imprescindible que se realicen acciones preventivas de la obesidad en los centros educativos, comunidades y sociedad en general, que promuevan valores de respeto, solidaridad, cooperación, entre otros, y que incentiven a las personas obesas (especialmente a las niñas) a participar activamente, de forma continua y sin temores, en el control de su obesidad.

Finalmente, ambos sectores deben organizar talleres creativos con la población estudiantil y sus familias para que estas personas se autoexaminen de forma crítica y contextualizada, y comprendan la importancia del autocuidado de la salud y del control del peso corporal, realizando prácticas de alimentación y de actividad física saludables. Este auto-convencimiento debe contemplar la reflexión de sus creencias, saberes, experiencias, temores y preocupaciones acerca de la obesidad.

\section{Referencias bibliográficas}

Carey, S. (1992). The Origin and Evolution of Everyday Concepts. Cognitive Models of Science. Minneapolis: Universidad de Minnesota Press.

Cea, M. A. (1998). Metodología cuantitativa. Estrategias y técnicas de investigación social. Madrid: Síntesis, S. A.

Cook, S. Weitzman, M. Auinger, P. Nguyen, M. Dietz, W. (2003). Prevalence of a metabolic syndrome phenotype in adolescents: findings from the third National Health and Nutrition Examination Survey. 1988-1994. Arch Pediatr Adolesc Med, 157(8), 821-7. 
De la Pienda, J. A. (1999). Filosofía de las creencias. Revista de Filosofía de la Universidad de Costa Rica, 92 (XXXVII), 239-48.

Dewey, J. (1989). Cómo pensamos. Nuestra exposición de la reflexión entre pensamiento reflexivo y proceso educativo. Barcelona: Paidós.

Díaz, A. (2006). Etnografía y técnicas de investigación antropológica. Guía didáctica. Madrid: UNED.

Dietz, W. H., Robinson, T. N. (1998). Use of body mass index (BMI) as measure of overweight in children and adolescents. J Pediatr, 132(2): 191-3.

Freedman, D. S., Dietz, W. H., Srinivasan, S. R., Berenson, G. S. (1997). The relation of overweight to cardiovascular risk factors among children and adolescents: the Bogalusa Heart Study. Pediatrics, 103(6): 1175-82.

Field, A. E., Cheung, L., Wolf, A. M., Herzog, D. B., Gortmaker, S. L., Colditz, G. A. (1999). Exposure to the mass media and weight concerns among girls. Pediatrics, 103(3): E36.

Figueroa-Colon, R., Franklin, F. A., Lee, J. Y., Aldridge, R., Alexander, L. (1997). Prevalence of obesity with increased blood pressure in elementary school-aged children. South Med J, 90(8): 806-13.

Frenk, J., Freika, T., Bobadilla, J. L., Stern, C., Lozano, R., Sepúlveda, J. (1991). La transición epidemiológica en América Latina. Bol Oficina Sanit Panam, 111 (6): 485-95.

Geertz, C. (1973). The Interpretation of Cultures. New York: Basic Books.

Hill, A. J., Franklin, J. A. (1998). Mothers, daughters and dieting: investigating the transmission of weight control. $\mathrm{Br}$ J Clin Psychol, 37(1): 3-13.

Hill, A. J., Silver, E. K. (1995). Fat, friendless and unhealthy: 9-year-old children's perception of body shape stereotypes. Int J Obes Relat Metab Disord, 19(6): 423-30.

Janssen, I., Craig, M., Boyce, W. F., Pickett, W. (2004). Associations between overweight and obesity with bullying behaviors in school-aged children. Pediatrics, 113(5): 1187-94.

McCabe, M. P., Ricciadelli, L. A. (2001). Parent, peer, and media influences on body image and strategies to both increase and decrease body size among adolescent boys and girls. Adolescent, 36(142): 225-40.

Madrigal, J. (1997). La construcción de indices. San José, Costa Rica: Editorial UCR.

Maxwell, J. (1996). Qualitative research design: An interactive approach. USA: Sage Publications, Inc.

Ministerio de Educación Pública. (2004). Transversalidad en el Currículo Educativo Costarricense, Relanzamiento de la Educación Costarricense. Costa Rica: Comisión Nacional de Transversalidad, Ministerio de Educación Pública.

Ministerio de Salud. (1996). Encuesta Nacional de Nutrición. Fascículo 1 Antropometría. Costa Rica: Ministerio de Salud.

Morrison, J. A., Barton, B. A., Biro, F. M., Daniels, S. R., Sprecher, D. L. (1999). Overweight, fat patterning and cardiovascular disease risk factors in black and white boys. J Pediatr, 135 (4): 451-7. 
Muris, P., Meesters, C., Van de Blom, W., Mayer. B. (2005). Biological, psychological, and sociocultural correlates of body change strategies and eating problems in adolescent boys and girls. Eat Behav, 6(1):11-22.

Max-Neff, M. (1986). Desarrollo a Escala Humana. Una opción para el Futuro. Chile: CEPAUR.

Núñez, H. P., Monge, R., Elizondo, A. M. (2003). Memoria del taller sobre calidad de la dieta de población escolar y adolescente. Tres Ríos, Costa Rica: INCIENSA, 10-5.

Pinhas-Hamiel, O., Dolan, L. M., Daniels, S. R., Standford, D., Khoury, P. R., Zeitler, P. (1996). Increased incidence of noninsulin dependent diabetes mellitus adolescents. J Pediatr, 128(5): 608-15.

Pozo, J. (1996). Aprendices y maestros. Madrid: Alianza.

Presnell, K., Bearman, S. K., Stice, E. (2004). Risk factors for body dissatisfaction in adolescent boys and girls: a prospective study. Int J Eat Disord, 36(4): 389-401.

Rodríguez, G., Gil, J. y García, E. (1996). Metodología de la investigación cualitativa. Málaga: Ediciones Ajibe.

Rogoff, B. (2002). Aprendices del pensamiento: El desarrollo cognitivo en el contexto social. Barcelona: Paidos.

Schumann, S. (1999). Creencias que manejan las docentes y los alumnos en el salón de clases. Tesis de Licenciatura para la obtención del título de
Licenciado en Psicología, Universidad Nacional, Heredia, Costa Rica.

Solano, M. (1987). La psicología de las clases sociales. San José, Costa Rica: Editorial Alma Máter.

Strauss, R. S. (2000). Childhood obesity and self-esteem. Pediatrics, 105(1):e15.

Strauss, R. S., Pollack, H. A. (2003). Social marginalization of overweight children. Arch Pediatr Adolesc Med, 157 (8): 746-52.

Vigotsky, L. (1978). Pensamiento y Lenguaje. Buenos Aires, Argentina: La Pleyade.

Weiss, R., Dziura, J., Burgert, T. S. (2004). Obesity and the metabolic syndrome in children and adolescents. $N$ Engl $J$ Med, 350(23): 2362-74.

Williams, C. L., Gulli, M. T., Deckelbaum, R. J. (2001). Prevention and Treatment of Childhood Obesity. In. CRC Handbook of Nutrition.

World Health Organization. (1995). Physical status: The use and interpretation of anthropometry. WHO Tech. Rep. Series 854: Switzerland: WHO.

World Health Organization. (2000). Obesity: preventing and managing the global epidemic. Geneva: WHO. (WHO Technical Report Series 894).

Zalilah, M. S., Anida, H. A., Merlin, A. (2003). Parental perceptions of children's body shapes. Med J Malaysia, 58(5): 743-51. 